\title{
Frontières
}

\section{L’annonce d'une mauvaise nouvelle en médecine}

\section{Roger Ladouceur}

Volume 14, numéro 2, printemps 2002

La mort prononcée

URI : https://id.erudit.org/iderudit/1073974ar

DOI : https://doi.org/10.7202/1073974ar

Aller au sommaire du numéro

Éditeur(s)

Université du Québec à Montréal

ISSN

1180-3479 (imprimé)

1916-0976 (numérique)

Découvrir la revue

Citer cet article

Ladouceur, R. (2002). L’annonce d'une mauvaise nouvelle en médecine. Frontières, 14(2), 66-69. https://doi.org/10.7202/1073974ar

\section{Résumé de l'article}

Cet article veut définir ce qu'est une mauvaise nouvelle en médecine, expliquer pourquoi il est si difficile pour un médecin de l'annoncer et présenter le programme Comment annoncer une mauvaise nouvelle élaboré par le Collège des médecins du Québec et le Collège québécois des médecins de famille. d'utilisation que vous pouvez consulter en ligne.

https://apropos.erudit.org/fr/usagers/politique-dutilisation/ 


\section{Résumé}

Cet article veut définir ce qu'est une mauvaise nouvelle en médecine, expliquer pourquoi il est si difficile pour un médecin de l'annoncer et présenter le programme Comment annoncer une mauvaise nouvelle élaboré par le Collège des médecins du Québec et le Collège québécois des médecins de famille.

Mots clés : médecin - mauvaise nouvelle - peur - formation continue

\section{Abstract}

This article acts as a definition of what bad news means in medicine and as an explanation of why it is so difficult for a doctor to break it and show the program How to Break Bad News put together by the Québec Doctors' College and the Québec College of Family Doctors.

Key words: physician - bad news - fear continuing education

\section{L'ANNONCE D'UNE MAUVAISE NOUVELLE
EN MÉDECINE NOUVELLE
EN MÉDECINE} professeur adjoint de clinique, Département de médecine familiale,Université de Montréal.

Annoncer une mauvaise nouvelle fait partie du travail quotidien du médecin. Ce n'est jamais facile. Si, pour tous et chacun, c'est difficile en raison des réactions qu'on appréhende comme la stupéfaction, l'incrédulité, la négation, la colère ou le désarroi, pour un médecin ce n'est guère plus aisé. Faire venir des parents à l'urgence pour leur apprendre que leur enfant vient d'avoir un grave accident ou annoncer à quelqu'un que ses résultats d'examens pour le sida sont positifs constitue une tâche pénible pour un médecin. Cette situation lui fait vivre bien
Roger Ladouceur, M.D., M.Sc., FCMC(C), médecin de famille, C.H. Verdun, des tourments : la peur d'être blâmé en tant que porteur de la mauvaise nouvelle, la peur de susciter des réactions négatives ou inattendues, la peur d'être confronté à son impuissance et à ses limites, la peur de l'inconnu et du non-dit, la peur de ses propres émotions et aussi sa propre peur. Pas surprenant dès lors que les médecins considèrent que dire la vérité et savoir comment la dire font partie des problèmes éthiques les plus fréquents (Brody et Tomlinson, 1988). Pas surprenant non plus que l'annonce d'une mauvaise nouvelle fasse l'objet de tant de doléances et de plaintes de la part des patients ou de leurs familles qui se disent insatisfaits de la façon de faire du médecin (Mongeon, Foulcault et Ladouceur, s.d.).
Ce soir-là, la veille de Noël, Martin n'en pouvait plus et il a décidé d'aller à l'urgence. Quelque chose n'allait plus avec sa santé. À 31 ans, ce n'est pas normal de toujours se sentir si fatigué, de devoir aller se coucher à tout moment de la journée puis de se lever tout aussi épuisé. Et de perdre du poids sans raison. Il n'avait plus faim. Son teint s'était mis à changer et son ventre à enfler. Il avait voulu en parler à un médecin, mais il n'avait pu avoir de rendez-vous avant quelques mois. Aussi, lorsqu'il s'est mis à avoir mal au ventre et à uriner du sang, il s'est dit qu'il fallait qu'il aille à l'urgence.

Ce soir-là, la veille de Noël, le docteur Brazeau était de garde. Lorsqu'il a aperçu Martin, il s'est dit que quelque chose n'allait pas: les traits tirés, le teint pâle et terreux, les conjonctives jaunes, le ventre enflé et ballonné, la fonte musculaire. Quelque chose de sérieux minait ce grand jeune homme. Il prescrivit une série de tests hématologiques et biochimiques. Il demanda aussi une échographie hépatique et une tomodensitométrie abdominale. Les examens confirmèrent rapidement ses présomptions.

Ce soir-là, Martin ne s'attendait certainement pas à ce qu'on lui annonce qu'il avait un cancer avancé; ce soir-là, le docteur Brazeau ne s'attendait pas à devoir le lui annoncer. Il se demandait bien ce qu'il allait lui dire et comment le lui dire. 
Cet article aborde les problèmes que soulève l'annonce d'une mauvaise nouvelle en médecine. Après avoir défini ce qu'est une mauvaise nouvelle, nous essayons de comprendre pourquoi il est si difficile pour un médecin d'en faire l'annonce et nous présentons un atelier de formation médicale continue réalisée par deux collèges de médecins au Québec.

\section{QU'EST-CE QU'UNE}

\section{MAUVAISE NOUVELLE ?}

Essayons d'abord de comprendre ce qu'est une mauvaise nouvelle. Se faire dire qu'on doit être opéré, qu'on vient de faire une fausse couche ou qu'on souffre d'un cancer généralisé, ce ne sont pas là de bien bonnes nouvelles! Mais qui sait? Peut-être que cette opération va permettre de trouver une solution à des douleurs insoutenables, que cette grossesse n'était pas désirée ou même que ce cancer sera reçu comme une bénédiction du ciel venant mettre fin à une vie remplie de souffrances. D'ailleurs, n'entend-on pas souvent les personnes âgées exprimer leur ouverture à la mort en disant : «Le bon Dieu m'a oublié. » Ainsi, une même information peut être perçue fort différemment d'une personne à l'autre, l'un la jugeant mauvaise et l'autre bonne. C'est vraiment dans la tête de celui qui la reçoit et de celui qui la donne qu'une nouvelle apparaît comme étant bonne ou mauvaise. C'est donc la perception que se font les individus d'une information qui la rend soit bonne ou mauvaise. Buckman (1984) définit de la façon suivante la mauvaise nouvelle en médecine: " une mauvaise nouvelle est toute information qui risque de changer complètement la perception que se fait un individu de son avenir, soit au moment du diagnostic ou lors d'un échec thérapeutique ». Il ajoute : "une même nouvelle peut être perçue plus ou moins mauvaise, selon les attentes du patient, son état actuel et l'intuition qu'il a de sa condition et de sa maladie».

\section{POURQUOI EST-IL SI DIFFICILE POUR UN MÉDECIN D'ANNONCER UNE MAUVAISE NOUVELLE ?}

S'il est difficile pour quiconque d'annoncer une mauvaise nouvelle, cela l'est tout autant, sinon plus, pour un médecin. En effet, annoncer une mauvaise nouvelle suscite chez lui plusieurs craintes et anxiétés qui sont propres à son rôle professionnel. Ces craintes sont les suivantes.

\section{LA PEUR D'ÊTRE BLÂMÉ EN TANT \\ QUE PORTEUR DE LA NOUVELLE}

La première peur qu'éprouve un médecin au moment de l'annonce d'une mauvaise nouvelle est certainement celle d'être personnellement blâmé pour ce qui arrive. C'est probablement l'une des pires inquiétudes et elle se manifeste même si le médecin n'a rien à se reprocher. Elle touche plus particulièrement le jeune médecin, mais aucun, même parmi les plus expérimentés, n'est à l'abri de cette réaction. Le fait d'être personnellement blâmé peut survenir à l'annonce de n'importe quelle mauvaise nouvelle, mais éclate surtout lorsque la nouvelle est inattendue et dramatique: la découverte subite d'une maladie incurable ou le décès inattendu d'un être cher transporté à l'urgence. Des phrases comme celles-ci traduisent bien le désarroi (et l'inquiétude) du médecin qui annonce la mauvaise nouvelle: "Je suis vraiment désolé, il n'y a rien d'autre à faire» ou «Nous avons tout essayé, mais sans succès ».

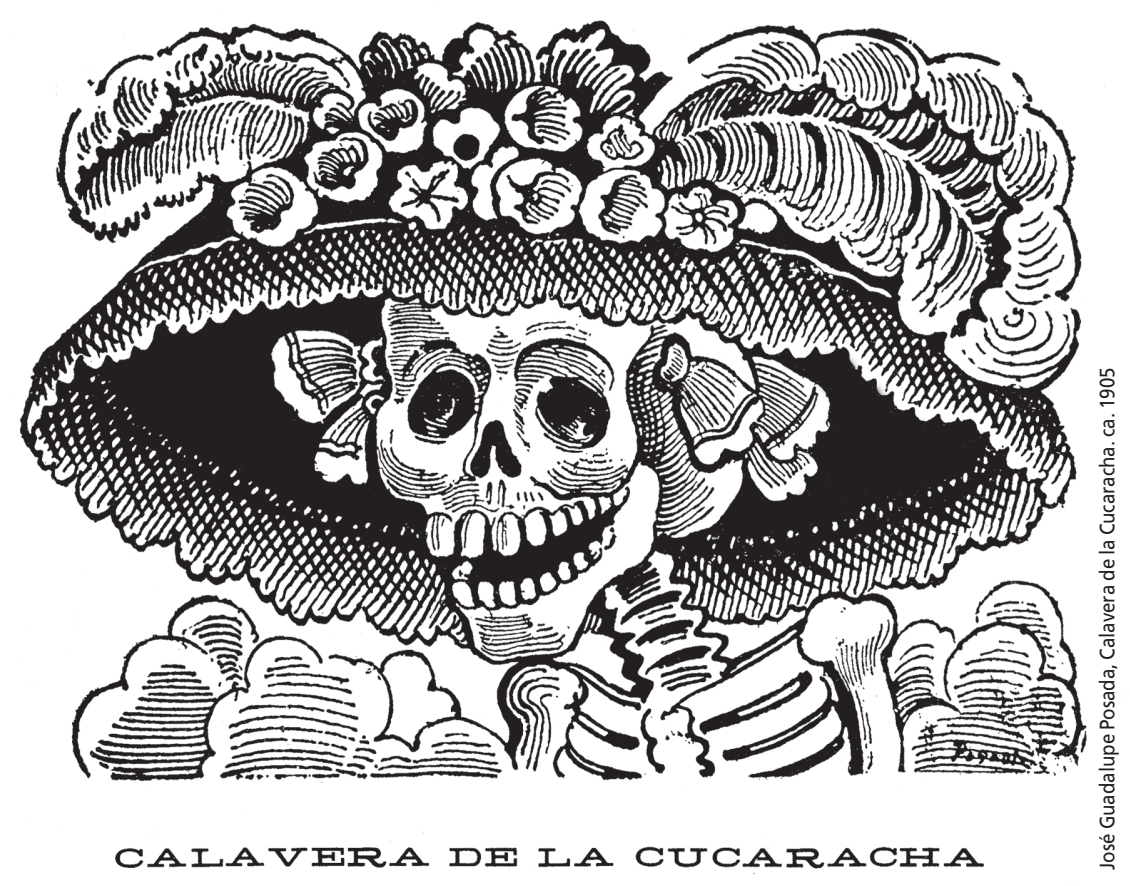

Évidemment, blâmer le messager n'est pas nouveau, ni propre à la médecine. Dans l'Antiquité, il arrivait que le messager d'une mauvaise nouvelle soit mis à mort. Dans la vie de tous les jours, on a tendance à blâmer régulièrement la personne par laquelle le malheur arrive : l'enseignant qui impose une pénalité pour un comportement inadéquat, le policier qui remet un billet pour un excès de vitesse, le juge qui rend un verdict de culpabilité. Mais pour le médecin, la situation a ceci de particulier qu'il est facile de le rendre responsable du malheur qui arrive. Et paradoxalement, plus le médecin est proche de son patient, plus il risque de subir l'odieux du reproche: "si je n'avais pas eu à attendre si longtemps avant d'avoir un rendez-vous! » ou «si vous m'aviez fait passer les bons examens plus rapidement!» ou « si vous me l'aviez dit plus tôt! ». Il est facile pour le patient ou pour sa famille de voir dans le médecin la personne à l'origine de tous les maux, surtout si ce dernier adopte une attitude paternaliste. N'a-t-il pas tous les pouvoirs : il décide du moment opportun pour faire passer les examens, il prescrit les médicaments à prendre ou à ne pas prendre, il rédige les références aux spécialistes? Comment se fait-il alors qu'avec tous les développements de la science, il ne soit pas capable de guérir ou de sauver?

Tous les patients ne réagissent pas de cette façon à l'annonce d'une mauvaise nouvelle, mais cela arrive suffisamment souvent pour que plusieurs médecins hésitent avant d'annoncer une mauvaise nouvelle, soit en remettant l'annonce à plus tard, en l'escamotant ou en tentant de refiler la responsabilité à un collègue, par exemple, en demandant une consultation en soins palliatifs.

\section{LA PEUR DE L'INCONNU}

Les médecins sont entraînés pour faire face à une multitude de problèmes médicaux. Ils apprennent à reconnaître, diagnostiquer et traiter la plupart des malaises et maladies. Ils sont capables de gérer diverses conditions, des plus simples aux plus complexes: les arrêts cardiorespiratoires, les comas ou les chocs nécessitant un transfert aux soins intensifs. Pour ce faire, ils s'appuient sur un enseignement qu'ils ont reçu dans des programmes de formation universitaire ou sur des évidences scientifiques rigoureuses.

Mais l'annonce d'une mauvaise nouvelle fait davantage appel à des habiletés de communication qu'à un ensemble de connaissances ou de compétences. Or, la plupart des médecins apprennent à annoncer une mauvaise nouvelle par eux-mêmes, et font de leur mieux, ou en observant un collègue qui l'a appris par lui-même. Comment annoncer une mauvaise nouvelle n'est pas enseigné systématiquement à l'université et 
il existe peu de documentation scientifique sur ce sujet et sur la meilleure façon de faire (voir How to Break Bad New, 1992). Leur façon de faire est donc empirique, personnelle, ne s'appuyant sur aucun schème de référence. Ce qui fait que plusieurs médecins s'interrrogent quant à la meilleure façon de procéder.

\section{LA PEUR DE PROVOQUER \\ DES RÉACTIONS INATTENDUES}

Évidemment annoncer une mauvaise nouvelle n'est pas de tout repos. Tout peut survenir. Annoncer une mauvaise nouvelle prend du temps. C'est difficile dans le cadre d'un bureau ou d'une urgence, avec un horaire surchargé, alors que déjà beaucoup de patients attendent depuis longtemps. Et quoi faire si la personne éclate en larmes ou n'arrive plus à se contrôler? Et que diront les autres? Or, les médecins sont réputés être capables de garder la situation sous contrôle

Plusieurs autres peurs et inquiétudes tenaillent le médecin au moment d'annoncer une mauvaise nouvelle: la peur d'exprimer ses émotions, la peur de ne pas savoir, ses propres peurs et angoisses face à la maladie et à la mort. Toutes ces raisons expliquent mais ne justifient certainement pas que le médecin soit maladroit, hésitant et même fuyant lorsque vient le temps d'annoncer à un patient une nouvelle qui risque d'être difficile à recevoir.

\section{PROGRAMME DE FORMATION MÉDICALE CONTINUE DU COLLÈGE DES MÉDECINS DU QUÉBEC ET DU COLLĖGE QUÉBÉCOIS DES MÉDECINS DE FAMILLE}

Pour aider les médecins à annoncer une mauvaise nouvelle, le Collège des médecins du Québec et le Collège québécois des médecins de famille ont élaboré un atelier intitulé: Comment annoncer une mauvaise nouvelle (Collège des médecins du Québec, 1997). Cet atelier s'inscrit dans une série d'activités de formation médicale continue visant à améliorer la communication entre le médecin et le patient. Les autres ateliers proposés sous le thème Questions d'attente, Questions d'entente, sont: le patient difficile, la bonne distance et l'observance thérapeutique.

L'atelier Comment annoncer une mauvaise nouvelle vise à aider le médecin à transmettre la nouvelle en utilisant une approche négociée et des moyens simples et en clarifiant le processus en cause. Les objectifs sont les suivants :

- Appliquer en entrevue les trois étapes de l'annonce d'une mauvaise nouvelle, soit l'avant, le pendant et l'après ;
- Acquérir des habiletés permettant d'enrichir, par divers moyens, des stratégies d'intervention dans ce type d'entrevue;

- Analyser, à l'aide d'une grille, la démarche de l'annonce d'une mauvaise nouvelle au cours d'une entrevue simulée.

L'atelier d'une durée de 90 minutes est offert gratuitement à tous les médecins du Québec. Il se déroule à la convenance des participants, habituellement sur leur lieu de travail. L'atelier est animé par un médecin de famille (trois médecins étant attitrés et formés) possédant une expertise en relation médecin-patient et en animation de petits groupes. Le Collèges des médecins du Québec et le Collège québécois des médecins de famille assument conjointement l'encadrement et l'organisation de l'activité. L'activité est soutenue et financée par la compagnie pharmaceutique Merck-Frosst Canada.

L'atelier utilise des mises en situation où les participants sont invités à annoncer une mauvaise nouvelle à un patient fictif, joué par l'animateur. Concrètement, les comportements recommandés pour annoncer une mauvaise nouvelle sont les suivants :

- Conditions : prévoir un temps raisonnable, utiliser un vocabulaire simple et accessible ;

- Avant d'annoncer la mauvaise nouvelle: préparer le terrain, choisir le bon moment, tenir compte du désir de savoir manifesté ou non par le patient;

- Au moment de l'annonce: doser l'information en fonction du patient, observer et nommer ses réactions, vérifier sa compréhension, lui demander d'exprimer ses sentiments ;

- Après avoir annoncé la mauvaise nouvelle: répondre en tenant compte des réactions du patient, garder l'espoir vivant.

Un document d'accompagnement, remis au participant, présente une synthèse de l'atelier et la documentation de référence. Un texte rédigé par Bigonnesse et Martel, omnipraticiens, sert d'assise aux recommandations principales.

\section{PRINCIPAUX ÉLÉMENTS DE L'ATELIER \\ La règle de base}

La règle du droit à l'information juste et complète constitue le principe de base permettant au patient d'exercer son autonomie et de participer pleinement aux prises de décision relatives à son contrat thérapeutique. Lorsqu'arrive le moment d'annoncer une mauvaise nouvelle, chacun a droit à une information juste et complète. De la sorte, le patient peut exercer son autonomie, exprimer ses choix et participer activement aux décisions thérapeutiques.

\section{Les stratégies spécifiques}

Les stratégies proposées sont simples et logiques et s'insèrent à différents moments de l'entrevue. Elles aident le médecin à orienter sa démarche.

Il est préférable, autant que possible, de connaître le patient ainsi que ses facultés d'adaptation dans des situations difficiles ou des crises antérieures. Idéalement, c'est le médecin traitant ou l'intervenant clé, et non pas un médecin consultant, qui annonce la nouvelle.

Avant tout.

1. Avoir du temps devant vous - Il faut éviter d'être dérangé (téléphone, télérécepteur). Il est préférable de s'asseoir près du patient et de maintenir un contact visuel. Le langage non verbal est tout aussi important que les mots.

2. Utiliser un vocabulaire simple, clair et accessible - Les médecins ont souvent tendance à utiliser une terminologie qui leur est propre. Des mots comme «métastases, fraction d'éjection, stade clinique» ne font pas partie du vocabulaire courant. Un langage simple et clair facilite la compréhension. Cependant, dans un contexte d'anxiété ou de détresse émotive, même un vocabulaire simple peut être mal interprété par le patient. Il importe donc au cours de l'entrevue, de vérifier la compréhension et de répéter au besoin l'information en changeant de vocabulaire.

Avant de procéder à l'annonce de la mauvaise nouvelle...

3. Préparer le terrain et prévenir le patient - Préparer le terrain consiste à amener le patient à découvrir progressivement son état. Habituellement, les patients se doutent bien que quelque chose ne va pas. L'une des façons de préparer le terrain est de revoir avec le patient l'évolution de sa condition. Les indices sont avancés progressivement pour lui permettre de cheminer, même au cours du premier entretien.

4. Choisir le moment optimal - Certains lieux et moments sont peu propices à l'annonce d'une mauvaise nouvelle: les corridors surpeuplés, les salles d'urgence bondées; les périodes qui suivent des examens exténuants ou douloureux. Une conversation téléphonique n'est jamais un bon moyen pour annoncer une mauvaise nouvelle, même si l'intention est de vouloir accélérer des rendez-vous ou des traitements (Premi, 1981; Miranda et Brody, 1992). Si vous sentez que le patient est fragile et si vous n'êtes pas sûr de ses réactions, il sera utile qu'un proche l'accompagne lors de l'annonce de la nouvelle pour lui apporter soutien et réconfort après la rencontre. 
5. Tenir compte de son désir de savoir La question de dire ou ne pas dire a longtemps fait l'objet de discussions et de controverses. Dans les années 1960, tout le dilemme était: dire ou ne pas dire... Dans les années 1970 et au début des années 1980, la société et la communauté médicale nord-américaines en sont arrivées à un consensus sur le droit de savoir du patient. Aujourd'hui, la plupart des personnes désirent savoir ce qui leur arrive, mais elles ne désirent pas l'apprendre n'importe comment; alors que d'autres ne désirent pas vraiment l'apprendre, en tout cas, pas tout de suite. Une question simple comme "J'ai eu les résultats de vos examens, désirez-vous les avoir? Je crains qu'ils ne soient pas très rassurants, voulez-vous que nous en parlions? » laisse la latitude voulue au patient pour exprimer son désir de savoir.

$\mathrm{Au}$ moment d'annoncer la mauvaise nouvelle...

6. Doser l'information - L'une des erreurs des médecins lorsque vient le temps d'annoncer une mauvaise nouvelle est de transmettre trop d'informations au patient dans l'espoir de le rassurer. Laisser le temps au patient de digérer la nouvelle. Une bonne façon de procéder pour doser l'information qu'on veut livrer est de lui demander où il en est à propos de son état: "Que savezvous au juste de ce qui se passe?» Pour certains auteurs, c'est le patient qui oriente le questionnaire et donne des indications sur les réponses qu'il est capable d'entendre. Il faut accepter de scinder l'information et de la divulguer par étapes, au cours de quelques visites. Si vous percevez une certaine réticence chez le patient, laissez-lui le temps de s'adapter au rythme auquel la nouvelle sera révélée. C'est le niveau d'anxiété du patient qui guide le médecin pour continuer ou non l'entrevue. Par exemple, une personne à qui l'on annonce qu'elle souffre d'un cancer et qui s'effondre, l'œil hagard, l'air absent, n'enregistre plus la moindre information. Il importe de la réconforter, de discuter de sa réaction et de remettre à plus tard la question du plan de traitement.

7. Observer les réactions du patient - Des questions simples et ouvertes permettent au médecin de mieux comprendre la réaction du patient, laquelle est fonction de sa perception du problème. On observe trois ordres de réactions: les réactions psychophysiologiques (agitation, activité psychomotrice accrue, effondrement avec retrait et mutisme), les réactions cognitives (déni, blâme, intellectualisation, incrédulité, acceptation) et les réactions affectives (colère, peur, anxiété, impuissance, désespoir, honte, culpabilité, délivrance). L’observation de ces réactions permet de situer le patient dans son évolution.

8. Vérifier si le patient comprend bien la situation - Les patients, pour la plupart, réagissent à l'annonce d'une mauvaise nouvelle en faisant référence à des expériences ou à des souvenirs semblables vécus par des proches ou véhiculés par les médias. Le rôle du médecin est de corriger les distorsions de la réalité qui peuvent alors survenir. Demander sim-plement au patient ce qu'il comprend de ce que vous lui dites permettra d'éviter ces mauvaises compréhensions.

9. S'intéresser aux sentiments du patient - Le patient qui s'effondre et se met à dramatiser tout comme celui qui accepte trop facilement une mauvaise nouvelle en posant des questions neutres sur des détails techniques concernant les traitements, sont à surveiller. Que se passe-t-il en eux? Quelles sont leurs émotions? Sont-ils en colère? désespérés? Que se passe-t-il à l'intérieur d'eux? Pourquoi ressentent-ils ces émotions?

Et finalement, une fois que l'annonce de la mauvaise nouvelle a été faite...

10.Garder l'espoir vivant - Même s'il peut sembler difficile de garder l'espoir vivant lorsqu'on annonce une mauvaise nouvelle, il s'agit là d'une dimension très importante. Peu importe l'ampleur de la mauvaise nouvelle, il importe de réconforter le patient tout en respectant sa peine, de le diriger vers les ressources dont il pourrait avoir besoin et de lui garantir votre soutien.

11.Répondre aux questions du patient Répondre à ses questions, respecter son besoin de silence ou de retrait et corriger les mauvaises interprétations.

\section{ÉVALUATION DU PROGRAMME}

L'atelier Comment annoncer une mauvaise nouvelle a connu un énorme succès au Québec. À ce jour, plus de 900 participants regroupés en plus de 70 ateliers y ont participé. Une évaluation (Ladouceur et al., s.d.) faite auprès des 706 participants, inscrits aux 50 ateliers ayant eu lieu entre septembre 1997 et juin 1998, a révélé qu'immédiatement après la rencontre, les éléments retenus sont les suivants : «tenir davantage compte du patient (44\%), mieux se préparer à la rencontre (12\%), améliorer ses habiletés de communication (10\%), prendre plus de temps et choisir un meilleur moment pour annoncer une mauvaise nouvelle». Six mois plus tard, un questionnaire envoyé aux mêmes participants montrent qu'ils retiennent presque les mêmes messages dans des proportions semblables: mieux tenir compte du patient (46\%), mieux préparer l'entrevue (10\%), améliorer les habitudes de communication $(14 \%)$ et prendre plus de temps ou choisir le meilleur moment pour annoncer une mauvaise nouvelle sont les messages retenus les plus souvent cités. Par contre, les difficultés qu'éprouvent les médecins sont la peur de la réaction du patient (17\% des énoncés), la gestion de ses propres émotions (9\%) et la peur de ne pas pouvoir consacrer suffisamment de temps à la rencontre $(7 \%)$. Les médecins ont déclaré que l'atelier avait été très aidant. Le fait de pouvoir discuter avec des collègues et les jeux de rôle sont jugés favorablement.

\section{Bibliographie}

BRODY, H. et T. TOMLINSON (1988). "On truth telling and the diagnosis of Alzheimer's disease (commentary). », J. Fam. Pract., vol. 26, n 4 , p. 401-406.

BUCKMAN, R. (1984). «Breaking bad news: why is it so difficult? ", B. Med. J., $\mathrm{n}^{\circ} 288$, p. $1597-1599$.

COLLÈGE DES MÉDECINS DU QUÉBEC (1997). Comment annoncer une mauvaise nouvelle, Programme conjoint du Collège des médecins du Québec et du Collège des médecins de famille du Canada, section Québec, sur la relation médecin-patient, Montréal.

How to Break Bad News - A guide for Health Care Professionals (1992). Baltimore, Johns Hopkins University Press, 223 p.

LADOUCEUR, R., R. GAGNON, F. GOULET, R. BOULÉ, G. GIRARD, A. JACQUES, R. CARRIER, V. LALONDE, J. FRENETTE et C. BÉLISLE (s.d.). L'annonce d'une mauvaise nouvelle: perception des médecins de l'impact d'un atelier de formation continue sur leur exercice professionnel, Direction de l'amélioration de l'exercice, Collège des médecins du Québec.

LIND, S.E., M. GOOD, S. SEIDEL, T. CSORDAS et B.J. GOOD (1989). "Telling the diagnosis of cancer», J. Clin. Oncol., $\mathrm{n}^{\circ}$ 7, p. 583-589.

MIRANDA, J. et R.V. BRODY (1992). "Communicating bad news", West J. Med., $\mathrm{n}^{\circ} 156$, p. 83-85.

MONGEON, S., C. FOULCAULT et R. LADOUCEUR (s.d.). Perception des aidants naturels face aux soins terminaux.

PREMI, J.N. (1981). "Communicating bad news to patients"Can. Fam. Physician, $\mathrm{n}^{\circ} 27$, p. $837-841$. 